Communications in Physics, Vol.18, No. 1 (2008), pp. 23-26

\title{
BRST CHARGE OPERATOR FOR GENERALIZED DEFORMED SU(2) ALGEBRA
}

\author{
NGUYEN THI HA LOAN \\ Hanoi Pedagogical University No 2 \\ NGUYEN HONG HA \\ Institute of Physics and Electronics VAST
}

\begin{abstract}
The BRST Charge plays a prominent role in interaction theory based on the Gauge symmetry group. In this work we find the explicit expression of the BRST charge for Generalized deformed $S U(2)$ algebra.
\end{abstract}

\section{INTRODUCTION}

The study of quantum groups and algebra [1] - [3] is a direction of actual character in theoretical physics. They find application in many problems such as quantum inverse scattering theory, exactly solvable model in statistical mechanics, rational Conformal field theory, two-demensional field theory with fractional statistics, etc . . .

The algebraic structure of quantum group can be formally described as a deformation, depending on one or more parameters, of the "classical" Lie algebras [4] - [5] . In the special limiting cases of these parameters the quantum algebras reduce to the ordinary Lie algebras. Especially the BRST formalism has proved to be powerful for the study of string theory

The subject of the paper is to find the explicit expression of BRST charge in generalized deformed group $S U_{\{q\}}(2)$

\section{MULTIPARAMETER DEFORMED $\mathrm{SU}_{\{q\}}(2)$}

1. The quantum algebra $S U_{\{q\}}(2)$ generated by the generators E, F, H obeys the commutation relations

$$
\begin{gathered}
E F-\beta(\{q\}) F E=f(H,\{q\}) \\
{[H, E]=2 E} \\
{[H, F]=-2 F}
\end{gathered}
$$

$\beta$ - some function of $\{q\}, f$ - some function of $H$ and $\{q\}$.

The Casimir operator $C$ obeys commutation relations

$$
[C, E]=[C, F]=[C, H]=0
$$

Its explicit expression is found to be

$$
C=\beta^{\frac{1}{2} H}\left\{E F+\beta^{-1} \phi\left(\frac{1}{2} H\right) \phi\left(\frac{1}{2} H-1\right)\right\}
$$

$\phi(x)$ - some function satisfying the relation:

$$
\phi(x-1)\{\beta \phi(x)-\phi(x-2)\}=\beta f(2 x-2) .
$$


2. In the limiting case $\beta(\{q\})=1, f(x,\{q\})=x, \phi(x)=x$ expression (1) gives back the familiar expression known in the ordinary algebra $\mathrm{SU}(2)$

$$
\begin{gathered}
{[E, F]=H} \\
{[H, E]=2 E} \\
{[H, F]=-2 F}
\end{gathered}
$$

The Casimir operator $\mathrm{C}$ is

$$
\begin{aligned}
& C=E F+\frac{1}{2} H\left(\frac{1}{2} H-1\right) \\
& =E F+\frac{1}{4} H^{2}-\frac{1}{2} H \\
& =I_{1}^{2}+I_{2}^{2}+I_{3}^{2}
\end{aligned}
$$

where

$$
\begin{aligned}
& E=I_{1}+i I_{2} \\
& F=I_{1}-i I_{2} \\
& H=2 I_{3}
\end{aligned}
$$

3. The representation of quantum algebra $S U_{\{q\}}(2)$

In the Hilbert space with the basic $|j m\rangle$ with $j=0, \frac{1}{2}, 1, \frac{3}{2}, \ldots, m=j, j-1, \ldots,-j$ the action of the generators $E, F, H$ yields

$$
\begin{aligned}
& H|j m\rangle=2 m|j m\rangle \\
& E|j m\rangle=\left\{\beta^{j-m-1} \varphi(j-m) \varphi(j+m+1)\right\}^{\frac{1}{2}}|j, m+1\rangle \\
& F|j m\rangle=\left\{\beta^{j-m} \varphi(j-m+1) \varphi(j+m)\right\}^{\frac{1}{2}}|j, m-1\rangle
\end{aligned}
$$

$\varphi(x)$ - some function satisfying the equation

$$
\beta^{y}\{\varphi(y+1) \varphi(x)-\varphi(y) \varphi(x+1)\}=f(x-y) .
$$

Let us consider some special cases.

a) Multiparameter deformations

$$
f(x,\{q\})=\frac{\gamma^{x}-(\gamma \beta)^{-x}}{\gamma-(\gamma \beta)^{-1}}
$$

From (4), (9) we can prove that

$$
\phi=\varphi=f .
$$

b) One parameter deformation

$$
f(x,\{q\})=[x]_{q}=\frac{q^{x}-q^{-x}}{q-q^{-1}} .
$$

This corresponds to the values $\beta=1, \gamma=q$.

The Casimir operator is

$$
C=E F+\left[\frac{1}{2} H\right]_{q}\left[\frac{1}{2} H-1\right]_{q} .
$$

It can be shown that its eigenvalue is

$$
C=[j]_{q}[j+1]_{q}
$$

c) Two- parameter deformation

$$
f(x,\{q\})=[x]_{p q}=\frac{q^{x}-p^{-x}}{q-p^{-1}}
$$

This corresponds to the values $\beta=q^{-1} p, \gamma=q$. 
The Casimir operator is

Its eigenvalue is

$$
C=\left(q^{-1} p\right)^{\frac{1}{2} H}\left\{E F+\left(q p^{-1}\right)\left[\frac{1}{2} H\right]_{p q}\left[\frac{1}{2} H-1\right]_{p q}\right\}
$$

$$
C=\left(p q^{-1}\right)^{j}[j]_{p q}[j+1]_{p q}
$$

\section{OSCILLATOR REPRESENTATION}

Let us consider the operators $a_{1}, a_{2}$ and their adjoints $a_{1}^{+}, a_{2}^{+}$satisfying the algebraic relations

$$
\begin{aligned}
& a_{i} a_{i}^{+}-(\beta S)^{-1} a_{i}^{+} a_{i}=S^{N_{i}} ; i=1,2 \\
& {\left[a_{i}, a_{j}\right]=0 ; i \neq j}
\end{aligned}
$$

where $N_{i}$ are oscillator number operators which are defined from the $a_{i}, a_{i}^{+}$as follows

$$
\begin{aligned}
& f\left(N_{i},\{q\}\right)=a_{i}^{+} a_{i} \\
& f\left(N_{i}+1,\{q\}\right)=a_{i} a_{i}^{+} ; i=1,2
\end{aligned}
$$

as a result, from these relations we obtain

$$
\begin{aligned}
& {\left[N_{i}, a_{j}\right]=-a_{i} \delta_{i j}} \\
& {\left[N_{i}, a_{j}^{+}\right]=a_{i}^{+} \delta_{i j} .}
\end{aligned}
$$

$\mathrm{S}$ - some function of $\{q\}$ satisfying the equation

$$
\begin{gathered}
\beta^{y}\left\{S^{y} f(x,\{q\})-S^{x} f(y,\{q\})\right\}=f(x-y,\{q\}), \\
a_{i} a_{i}^{+}-x a_{i}^{+} a_{i}=\left[(\beta S)^{-1}-x\right] f\left(N_{i}\{q\}\right)+S^{N_{i}}
\end{gathered}
$$

for arbitrary $x$.

The generators of $S U_{\{q\}}(2)$ can be expressed in terms of two independent oscillators $a_{1}, a_{2}$ and their adjoints $a_{1}^{+}, a_{2}^{+}$as follows:

$$
E=a_{1}^{+} \beta^{\frac{N_{2}}{2}} a_{2}, F=a_{2}^{+} \beta^{\frac{N_{2}}{2}} a_{1}, H=N_{1}-N_{2}
$$

For two - parameter $(p, q)$ deformation $S=q, \beta=\left(p q^{-1}\right)$ and relations (18) and (23) reduce to the following relations:

$$
\begin{gathered}
a_{i} a_{i}^{+}-p^{-1} a_{i}^{+} a_{i}=q^{N_{i}}, \\
E=a_{1}^{+}\left(q^{-1} p\right)^{\frac{N_{2}}{2}} a_{2}, F=a_{2}^{+}\left(q^{-1} p\right)^{\frac{N_{2}}{2}} a_{1}, H=N_{2}-N_{1} .
\end{gathered}
$$

\section{THE BRST CHARGE IN GENERALIZED DEFORMED GROUP$$
S U_{\{q\}}(2)
$$

We introduce double ghosts $C_{l}(x)$ and antighosts $b_{l}(x)$ associated to the generators $E, F, H$ of deformed algebra (1). They obey the anticommutation relations

$$
\begin{aligned}
& \left\{C_{+}, C_{-}\right\}_{\beta}=0,\left\{b_{+}, C_{-}\right\}=1 \\
& \left\{C_{+}, C_{0}\right\}_{=} 0,\left\{b_{-}, C_{+}\right\}=1 \\
& \left\{C_{-}, C_{0}\right\}_{=} 0,\left\{b_{0}, C_{0}\right\}=1 \\
& \left\{b_{+}, C_{+}\right\}_{\beta}=0,\left\{b_{l}, b_{m}\right\}=0,(l, m= \pm, 0) \\
& \left\{b_{-}, C_{-}\right\}_{\beta^{-1}}=0 \\
& \left\{b_{0}, C_{-}\right\}_{\beta^{-1}}=0
\end{aligned}
$$


where we denote

$$
\{A, B\}_{f}=A B+f B A .
$$

From them explicit expression of the BRST charge $Q$ is found:

$$
\begin{aligned}
Q_{\{q\}}= & E C_{-}+F C_{+}+f(H,\{q\}) C_{0}+\beta^{-\frac{1}{2}} b_{0} C_{+} C_{-} \\
& +\frac{1}{2}[f(H+2,\{q\})-f(H-2,\{q\})]\left[b_{+} C_{-} C_{0}-b_{-} C_{+} C_{0}\right] \\
& +\frac{1}{2}[f(H+2,\{q\})+f(H-2,\{q\})-2 f(H,\{q\})] \\
& \times\left[b_{+} C_{-} C_{0}+b_{-} C_{+} C_{0}-2 b_{+} b_{-} C_{+} C_{-} C_{0}\right]
\end{aligned}
$$

For the case

we have

$$
f(x,\{q\})=[x]_{q}=\frac{q^{x}-q^{-x}}{q-q^{-1}}
$$

$$
\begin{aligned}
Q= & E C_{-}+F C_{+}+[H]_{q} C_{0}+b_{0} C_{+} C_{-} \\
& +\left(q^{H+1}+q^{-H-1}\right) b_{+} C_{-} C_{0}-\left(q^{H-1}+q^{-H+1}\right) b_{-} C_{+} C_{0} \\
& -\left(q-q^{-1}\right)^{2}[H]_{q} b_{+} b_{-} C_{+} C_{-} C_{0} .
\end{aligned}
$$

For the case

we have

$$
f(x,\{q\})=[x]_{p q}=\frac{q^{x}-p^{-x}}{q-p^{-1}}, \quad \beta=q^{-1} p
$$

$$
\begin{aligned}
& Q=E C_{-}+F C_{+}+[H]_{p q} C_{0}+\left(q^{-1} p\right)^{-\frac{1}{2}} b_{0} C_{+} C_{-} \\
& +\frac{q^{H}\left(q^{2}-1\right)+p^{-H}\left(1-p^{-2}\right)}{q-p^{-1}} b_{+} C_{-} C_{0} \\
& -\frac{q^{H}\left(1-q^{-2}\right)+p^{-H}\left(p^{2}-1\right)}{q-p^{-1}} b_{-} C_{+} C_{0} \\
& -\frac{1}{q-p^{-1}}\left\{\left(q-q^{-1}\right)^{2} q^{H}-\left(p-p^{-1}\right)^{2} p^{-H}\right\} b_{+} b_{-} C_{+} C_{-} C_{0}
\end{aligned}
$$

\section{ACKNOWLEDGMENTS}

Thanks are due to Prof. Dao Vong Duc for suggesting the problem and the fruitful discussion.

\section{REFERENCES}

[1] S. Woronowics, Comm. Math. Phys. 111 (1987) 613; V. G. Drinfeld, Sov. Math. Dokl. 32 (1985) 254; M.Jimbo, Lett. Math. Phys. 10 (1985) 63.

[2] L. Brink, T. H. Hansson and M. A. Vasiliev, Phys. Lett. B286 (1992) 109.

[3] M. Chichian, P. Kulish and J. Lukierski, Phys. Lett. B237 (1990) 401.

[4] L. V. Dung, N. T. H. Loan, Comm. in Phys. 4 (1994) 85-89.

[5] D. V. Duc, Generalized Multiparameter Quantum Algebra $S U_{\{q\}}(2)$, Preprint VITP 93- 08, Hanoi.

[6] D. V. Duc, Quantum Group Approach to Symmetry Breaking, Preprint VITP 92- 07, Hanoi.

Received 17 January 2008. 
